\title{
VALORES DE LA CLÍNICA. UNA INVITACIÓN A SABOREAR LA VIDA ${ }^{1},{ }^{2}$
}

\author{
Sandra Buechler 3
}

\section{Los valores vivos en el tratamiento}

Los valores que ponemos en juego al hacer el tratamiento es el tema de mi primer libro (2004), Valores de la Clínica. Creo que la forma en que vivimos nuestros valores, especialmente en aquello en lo que elegimos enfocarnos en una sesión, y en lo que no nos enfocamos, es crucial para el impacto que tenemos en los pacientes. Por lo tanto, estoy reinterpretando el tema de la transferencia y la contratransferencia, para incluir los valores que viven los dos participantes.

Fundamentalmente, veo el tratamiento como un diálogo sobre lo que significa vivir. El diálogo del tratamiento versa sobre lo que significa ser un ser humano y vivir una vida humana. Aunque estoy seguro de que sería posible enfocarme en otros aspectos de la humanidad, he descubierto que mis propios diálogos de tratamiento exploran con mayor frecuencia cómo las personas pueden modificar sus sentimientos más dolorosos accediendo a la curiosidad, la esperanza, la bondad, el coraje, el sentido de propósito, el equilibrio emocional, la capacidad de soportar la pérdida y la integridad. Estos problemas se repiten para mí. Las emociones fundamentales como la ira, la vergüenza y la alegría son el corazón de cada uno de estos desafíos humanos. Tomemos la capacidad de soportar la pérdida como un ejemplo. Creo que en el tratamiento vivimos este problema juntos. Inevitablemente lo encontramos de muchas maneras, especialmente al finalizar, pero también en otros

\footnotetext{
${ }^{1}$ Texto elaborado para el Seminario "Renovadores de la Psicoterapia Psicoanalítica" y leído por la autora el 3 de Octubre de 2018 en Ágora Relacional, Madrid, con la asistencia de los integrantes del seminario en sus convocatorias 2017/18 y 2018/19, con ocasión de la publicación de la versión castellana de su libro "Valores de la Clínica". Traducción castellana de Alejandro Ávila, coordinador del Seminario.

${ }^{2}$ Referencia para citar este trabajo: Buechler, S. (2019). Valores de la Clínica. Una invitación a saborear la vida. Clínica e Investigación Relacional, 13 (1): 22-31. [ISSN 1988-2939] [Recuperado de www.ceir.info ] DOI: 10.21110/19882939.2019.130102

3 Sandra Buechler, Ph.D. (New York) es Analista Didacta y Supervisora en el William Alanson White Institute. Supervisora en el Programa postdoctoral y de Residentes del Columbia Presbyterian Hospital, así como en el Institute for Contemporary Psychotherapy. Entre sus obras: Valores de la Clínica: Emociones que guían el tratamiento psicoanalítico (2004, 2018); Marcando la diferencia en la vida de los pacientes: Experiencia emocional en el contexto terapéutico (2008, 2015); Permanezco ejerciendo: Las alegrías y angustías de una carrera clínica (2012); Comprendiendo y tratando pacientes en Psicoanálisis Clínico: Lecciones desde la Literatura (2015) y Reflexiones psicoanalíticas. Formación y práctica (2018).
}

CelR Vol. 13 (1) - Abril 2019 ISSN 1988-2939 - www.ceir.info

@ Derechos reservados/Copyright de Clínica e investigación Relacional y los autores. Prohibida la reproducción total o parcial sin autorización expresa. Este material es para uso científico y profesional exclusivamente y puede contener información clínica sensible. Los editores no se responsabilizan de los contenidos de los autores. Dirigir las consultas sobre derechos y autorizaciones a ceir@psicoterapiarelacional.es 
momentos. Tenemos un diálogo en el que, a veces, se habla, acerca de cómo ser humano implica soportar la pérdida. Trabajamos algo acerca de cómo cada uno puede acercarse a la pérdida. Por supuesto, cualquier cosa con la que trabaje involucra las emociones fundamentales que son esenciales para la experiencia humana. Soportar bien la pérdida es una tarea ineludible. Supone una exigencia elevada, que requiere la colaboración de todo el sistema de emociones discretas. Necesito estar emocionalmente equipado para enfrentarlo repetidamente en los tratamientos que realizo. Debo usar la fuerza que puedo derivar de todas mis otras emociones, para modular las inevitables penas del trabajo. Creo que la pérdida bien llevada se reduce radicalmente. Es decir, todas las pérdidas deberían limitarse a lo que no podemos evitar perder. Cuando alguien muere, la relación con la esencia de esa persona debe ser lo suficientemente fuerte como para hacer que la pérdida de su presencia real sea llevadera. El duelo se convierte así en un encuentro activo con el significado de una relación. Todas las emociones fundamentales pueden contribuir a un dolor modulado, es decir, un dolor que realmente se siente, pero también equilibrado por otros sentimientos. La ira ante la inevitabilida de la pérdida, e incluso una feliz celebración de los logros de esa vida, pueden desempeñar un papel. La pérdida siempre está con nosotros, una parte inevitable de la condición humana. La única pregunta no es si sucede, sino cómo la vivimos. El diálogo del tratamiento explora la cuestión de cómo vivir la pérdida. A veces, esta exploración es explícita, ya que generalmente está en la terminación, si el tratamiento ha podido establecer una conexión genuina. Pero a lo largo del trabajo se producen conversaciones más sutiles e implícitas sobre la pérdida, en las decepciones comunes que impregnan nuestra experiencia. Oportunidades perdidas, esperanzas, sueños, son inherentes a su tejido. El tratamiento puede ser, para ambos participantes, un laboratorio para refinar la capacidad de soportar la pérdida.

\section{Dificultades de los clínicos con el concepto de valores clínicos}

¿Cómo puedo describir los valores que traigo a mi trabajo como terapeuta orientado psicoanalíticamente? Por más de una razón, los analistas han recelado de declarar nuestros valores como parte de nuestra profesión. Durante años, nos sentimos obligados a luchar por una especie de "neutralidad" que parecía impedir la manifestación de valores. Queríamos evitar ser vistos como adoctrinadores, como parte de un culto o una religión. Si bien esto es comprensible, creo que ha dejado una especie de vacío espiritual. ¿Cómo podemos luchar apasionadamente en nombre de la vida, si no podemos permitirnos nombrar nuestras convicciones? 
Considero que los valores son inherentes al corazón de nuestra profesión. Amamos y alimentamos nuestras propias aventuras intelectuales y la curiosidad en nuestros pacientes. Creemos en el poder de la esperanza, la bondad, el coraje, el sentido de propósito y la integridad. Sabemos que es parte de nuestro trabajo ayudar a las personas a ser más capaces de soportar las pérdidas y ser más capaces de mantener el equilibrio emocional. Probablemente sea una atracción por estos valores lo que nos ayudó a decidirnos a formarnos en esta profesión en primer lugar, pero luego esta tendencia se vio reforzada en nuestros años de formación. Hacemos todo lo posible para ayudar a las personas a llevar vidas mejores y más ricas. Creemos que la curiosidad cultivada, junto con el coraje de enfrentar verdades difíciles, puede ayudar a las personas a vivir más plenamente. $Y$ no estaríamos aquí si no quisiéramos ayudar a las personas a vivir más plenamente.

\section{Valentía en el tratamiento}

Uno de los valores más importantes que require el hacer tratamiento es el coraje o valentía. En el siglo IV, a.C., Aristóteles definió el coraje como la observancia de la media entre el miedo excesivo y la temeridad excesiva. Continúa diciendo que debemos valorar especialmente la valentía en respuesta a eventos repentinos, ya que la valentía "en un peligro imprevisto surge más del carácter, ya que hay menos tiempo para la preparación; uno puede decidir enfrentar un peligro que uno puede prever, por cálculo y por principio, pero solo una disposición fija de coraje le permitirá a uno enfrentar un peligro repentino ".

Sugiero que los terapeutas se enfrentan a la posibilidad del peligro cada sesión, por lo que debemos nutrir esa disposición fija de coraje en nosotros mismos. En Valores de la Clínica, nombro los peligros que creo que enfrentamos regularmente, y describo la timidez temeraria que siento que necesitamos cultivar.

Un importante acto de coraje es la decisión de convertirse en clínico. Esto requiere una timidez precipitada y un abrazo voluntario de muchas paradojas. Con algunos pacientes podemos ser los extraños más íntimos imaginables. Nos preocupamos profundamente por ellos, pero somos pagados por cada sesión. Entendemos nuestra subjetividad, que nunca podemos, como Steve Mitchell solía decir, ver fuera de la perspectiva moldeada por nuestra contratransferencia particular. Sin embargo, cuando un paciente nos cuenta una historia, nos damos cuenta de que lo que cuestionamos, lo que olvidamos, lo que escuchamos y respondemos, lo que saludamos, lo que recordamos la próxima sesión, puede tener algún impacto en la vida de ese paciente. Convertirse en un clínico significa, para mí, abrazar la paradoja de ser consciente de mis limitaciones $y$, sin embargo, seguir adelante con el trabajo clínico. 
Nuestras perspectivas son subjetivas en forma inherente y perpetua. Ninguna conducta en particular confirma la valentía del analista. La valentía analítica establece una atmósfera palpable de profunda libertad de expresión. Es el sentimiento de estar con la niña que en cualquier momento puede decir que el rey no lleva ropas encima. En la compañía de esta niña, cualquier cosa puede pasar. Ningún pensamiento es tabú, y todo lo que se puede enseñar se puede decir. Pero esta niña no perderá su sensación de ser ella misma si elige no señalar la desnudez del rey. En su presencia, siempre existe la posibilidad, pero no la certeza, de dar voz a lo que no se había dicho (Buechler, 2004, p. 83).

\section{Sentido de propósito y esperanza}

Otros valores que el trabajo clínico requiere incluyen tener un sentido de propósito y esperanza. Por ejemplo, el sentido de propósito que Fromm fomenta en el analista puede proporcionar un modelo al paciente de cómo los compromisos emocionales básicos pueden informar la vida laboral de uno, de modo que el trabajo se convierta en expression de uno mismo. En otras palabras, si un ser humano, analista o no, nutre activamente sus tendencias biofílicas, estas fuerzas encontrarán expresión en su trabajo, así como en las otras esferas de su vida. Un analista biofílico demuestra cómo el amor por la vida puede impregnar el trabajo, dándole significado y propósito. Para mí, el sentido de propósito no es un objetivo único, sino la sensación de que el trabajo es significativo y tiene el potencial de enriquecer dos vidas.

1. Para mí hay un significado inherente en la vida del paciente, y por lo tanto, siento un sentido de propósito al trabajar para mejorar la calidad de esa vida.

2. Estoy dispuesta a luchar por la vida del tratamiento, porque es significativo para mí.

3. La salud general del tratamiento es más significativa que mi orgullo, si tengo que escoger entre ellos.

4. La introspección significativa es algo de lo cual estar orgullosa a pesar de lo que revele.

5. No estoy avergonzada de mi fuerza de propósito. Querer apasionadamente que el paciente encuentre una vida significativa no es una fuente de conflicto para mí.

En cómo vivimos la sesión, transmitimos lo que encontramos significativo y lo que nos da un sentido de propósito. Mucho de esto se expresa en nuestro tono, más que en nuestra elección de las palabras. El tono puede manifestar pasión acerca del trabajo. A menudo he experimentado el tratamiento como una clase de lucha por la vida (Buechler, 2004, pp.9192). 
Creo que, especialmente en su último trabajo, Fromm entendió esta orientación activa hacia la vida como nuestra mejor fuente de esperanza sobre el futuro de la humanidad. La esperanza proviene de una dedicación inquebrantable a ser, en lugar de tener. La esperanza viene de trabajar duro por algo que realmente crees que vale la pena.

Encuentro que es esperanzador creer que las personas pueden expresar sus anhelos reales en su trabajo, en lugar de que se nos pueda sobornar para que se desempeñen, o esforzarnos para realizar obsequios o signos de aprobación. La esperanza de Fromm es práctica, activa, abrazando las paradojas de la vida. Aquí están sus propias palabras para eso.

Tener esperanza significa atreverse, pensar lo impensable $y$, con todo, actuar dentro de los límites de lo realistamente posible; la paradoja de la esperanza consiste en esperar al Mesías cada día y, sin embargo, no descorazonarse si éste no llega a la hora señalada. Esta esperanza no es pasiva y tampoco es paciente; por el contrario, es impaciente y activa, busca toda posibilidad para la acción dentro del campo de las posibilidades reales. (Erich Fromm, 1973, citado en Hornstein, 2000, p. 390; a su vez en Buechler, 2004, pp. 45-46)

¿Cómo inspiramos a nuestros pacientes a tener esperanza? ¿Y cómo mantenemos la esperanza viva en nosotros mismos? La esperanza, para mí, es más que una expectativa cognitiva de algo positivo. Es una emoción que nos motiva y nos impulsa a avanzar. En el tratamiento, la esperanza requiere abrazar la paradoja. Un paciente pregunta por qué debería creer que la terapia puede ayudarlo, cuando todavía está deprimido después de muchos años de tratamiento. Creo que, para los dos, mantener nuestra esperanza requiere aceptar verdaderamente la naturaleza paradójica del tratamiento $y$, más en general, de la vida misma. Cuando estoy muy agotada, gastar más energía para ejercitarme o escribir puede energizarme. A veces, el trabajo duro se actualiza. Esperar significa para mí tener la humildad de comprender que mis sentidos y mi lógica pueden confundirme. Lo inalterable puede cambiar. El trabajo duro puede actualizarse. Aceptar la profunda desesperación de un paciente puede levantarlo, porque entonces, al menos, no está solo.

La esperanza no es solo una idea, o una expectativa, sino una emoción activa y propulsora. La esperanza, como otros sentimientos, es un subproducto del equilibrio de todas las emociones. Al igual que la felicidad, no podemos aspirar conscientemente a la esperanza, pero si vivimos de manera saludable, tendremos esperanza. Alguna esperanza puede venir de apreciar la vida. La tarea de mantener la esperanza a menudo es, inicialmente, el trabajo del clínico. Debemos reconocer y trabajar en los obstáculos emocionales a la esperanza, en nosotros mismos, nuestros supervisados y nuestros pacientes.

Nuestra propia esperanza se transmite a través del trabajo duro y la perseverancia, incluso cuando nada parece estar cambiando. La falta de voluntad para darse por vencido envía un 
mensaje fuerte al paciente. Si estamos dispuestos a trabajar duro, incluso cuando no hay un resultado inmediato, y honestamente nos confrontamos a nosotros mismos, y enfrentamos el dolor, cuando es inevitable, estamos promulgando valores clínicos. Intenté describir cómo el médico inspira esperanza muchas veces. Aquí está una de las descripciones de cómo evocamos la esperanza.

Probablemente, el aspecto de la esperanza activa que afirma el compromiso con la vida no se transmite en el contenido de lo que se dice sino, más bien, en el fervor del tono, en el ímpetu de la convicción que puede ser indicado por la inmediatez y la vivacidad del habla. Pensándolo bien, probablemente se transmite al paciente más plenamente por las actitudes personales y profesionales que el analista revela sin darse cuenta. El amor al trabajo, la pasión por fomentar la vida y el crecimiento, una disposición enérgica hacia sí mismo y hacia los otros, la voluntad de luchar, el disfrute del humor y del desafío de la vida, son algunas de las cosas intangibles que se dan a conocer en el ritmo y las expresiones sutiles de la música, más que en las palabras. Aquello en lo que se enfoca el analista, a lo que responde, por lo que está dispuesto a romper el encuadre, lo que deja pasar en silencio, lo que enfrenta con pasión, lo que manifiesta en primera persona, lo que le agota, aquello contra lo que lucharía, dice muchísimo. Lo mismo puede decirse de la supervisión, donde las actitudes del supervisor y del supervisado en torno al tratamiento y la vida a menudo se encuentran, tanto explícita como implícitamente, bajo escrutinio [Buechler, 1995b, pp. 72-73; citado en Buechler, 2004, p.41].

\section{El amor a la verdad}

El trabajo clínico y la formación clínica deben manifestar otros valores, como la consistencia entre lo que decimos y cómo actuamos, o la integridad, y el respeto absoluto y el amor a la verdad, tal vez una verdad especialmente incómoda. Debemos amarlo por sí mismo, no como un medio para ningún fin. En la supervisión, especialmente cuando un candidato es lo suficientemente valiente como para decir la verdad sobre una situación clínica insatisfactoria, nuestra primera y más importante tarea es reconocer el coraje que exigió. No hay ninguna técnica, ninguna teoría más importante para enseñar que la adoración de la verdad, y un profundo respeto por cualquier persona dispuesta a contarla, especialmente en los momentos en que es inconveniente. La verdad incómoda está en el corazón mismo de los procesos analíticos y de supervisión. El valor de la bondad a veces se pasa por alto, pero creo que también tiene un lugar en el tratamiento. Entonces, en general, en la forma en que llevamos a cabo el tratamiento, debemos manifestar valor, sentido de propósito, esperanza, 
amor por la verdad y bondad, y nuestros programas de capacitación deben prepararnos para esta desafiante pero gratificante tarea.

\section{El valor de la colaboración analítica}

El análisis puede ser un encuentro verdaderamente vivo entre dos personas emocionalmente presentes y receptivas. Los momentos pueden ser emocionantes. Otros momentos pueden inquietarnos, aterrorizarnos, enfurecernos, tocarnos con tristeza. Pienso en la relación posible en el tratamiento como similar a la poesía en su plenitud destilada. Cada potencial de sentimiento en los seres humanos puede estar intensamente presente. Nuestra condición humana se manifiesta en forma pura, en este abrazo absurdamente limitado y gloriosamente ilimitado.

Juntos, siento que el analista y el paciente crean una relación de colaboración al estar dispuestos a contribuir con cualquier valor que puedan dar, al servicio de su trabajo. Si tengo una idea, si puedo hacer que la sesión sea más conveniente cuando el paciente está presionado por el tiempo, si puedo pasar algún tiempo estudiando la historia del paciente, o un concepto teórico potencialmente útil, creo que hacer estos esfuerzos puede alentar al paciente a participar completamente Estoy comunicando que vale la pena insistir en su interés. Estoy diciendo que importa a través de mis acciones, en lugar de palabras que pueden no ser muy convincentes. Otra forma en que he entendido la esperanza en ambos participantes del tratamiento (Buechler, 2004, 2008) es que es un producto del autoconocimiento. Si sé que haré todo lo que pueda para ayudar a un paciente, tengo motivos para estar esperanzado con el tratamiento.

\section{Disposición para esforzase}

La dedicación al trabajo no debe ser un principio abstracto, sino más bien un valor manifestado apasionadamente. La disposición a invertir esfuerzo se revela en lo difícil que tratamos de entender lo que el paciente está diciendo. ¿Estamos dispuestos a admitir que estábamos distraídos y le pedimos al paciente que repita lo que dijeron? ¿Dedicamos tiempo y nos preocupamos por conocer la historia del paciente y comprender su cultura cuando difiere de la nuestra? ¿Trabajamos en el uso de los sueños? ¿Nos esforzamos por ser conscientes de la contratransferencia y revelamos lo que creemos que favorecerá el tratamiento, ya sea que nos haga parecer competentes o no? Cuando hay una opción en cuanto a cuyo ego puede estar magullado, ¿qué hacemos? Por ejemplo, si hay un malentendido acerca del dia o la hora de la sesión, ¿qué reconocemos? 
La disposición a ser un "tonto por amor" (Buechler, 2004, pp. 58-60) ${ }^{1}$ es una de las bondades más esenciales que podemos desplegar en la terapia. Tomamos una posición acerca de lo que cuenta en el tratamiento $y$, de manera más general, en la vida, cuando exponemos voluntariamente nuestras propias limitaciones intelectuales y emocionales. Más que cualquier otra cosa, creo que esta disposición alienta al paciente a estar menos preocupado por su aspecto y más investido en conocerse a sí mismo y ser completamente conocido.

La vida es finita, preciosa, demasiado rica para ser desperdiciada. El tratamiento puede ayudarnos a saborearla. Tomo mucho significado del hecho de que la palabra "sabiduría" se deriva de "sapere" (Del latín, gustar, tener gusto, ser sabio: sabio, sapid, sapient, sapor, sabio, savor, experto. American Heritage Dictionary, 1969, p 1537). Como en la apreciación matizada de los detalles de un gourmet, saborea el sabor de la vida. Incluso el dolor, si se siente intensamente, nos dice que estamos vivamente vivos. Un tratamiento que ayuda a las personas a saborear la vida tiene un propósito intrínsecamente valioso.

\section{Referencias}

Aristotle (fourth century B.C.). The Nicomachean Ethics, trans. H. Rackham. Ware, Hertfordshire: Wordsworth Editions

Blechner, M.J. (1992). Working in the countertransference. Psychoanal. Dial, 2:161-179.

Blechner, M. (2001). The Dream Frontier. Hillsdale, NJ: The Analytic Press.

Buechler, S. (2004). Clinical Values. Emotions that guide psychoanalytic treatment. New York: Routledge

Buechler, S. (2008). Making a difference in patients' lives. New York: Routledge,

Coen, S. (2002). Affect Intolerance in Patient and Analyst. Northvale, NJ: Aronson.

Ehrenberg. D.B. (1992). The Intimate Edge: Extending the Reach of Psychoanalytic Interaction. New York: Norton.

Fromm, E. (1973). The Anatomy of Human Destructiveness. New York: Holt, Rinehart \& Winston.

Hirsch, I. (1995). Therapeutic uses of the countertransference. In: Handbook of Interpersonal Psychoanalysis, ed. M. Lionells, J. Fiscalini, C.H. Mann \& D.B. Stern. Hillsdale, NJ: The Analytic Press, pp. 643-661.

Hornstein, G.A. (2000). To Redeem One Person is to Redeem the World: The Life of Frieda Fromm-Reichmann. New York: free Pres

Maroda, K. (1999). Seduction, Surrender, and Transformation. Hillsdale, NJ: The Analytic Press. 
Maroda, K. (2002). No place to hide: Affectivity, the unconscious, and the development of relational techniques. Contemp. Psychoanal., 38:101-121.

\author{
Mitchell, S.A. (1993). Hope and Dread in Psychoanalysis. New York: Basic Books. \\ Mitchell, S.A. (1997). Influence and Autonomy in Psychoanalysis. Hillsdale, NJ: The Analytic \\ Press
}

Renik, O. (1998). Getting real in analysis. Psychoanal, Quart., 67:566-593.

\title{
NOTAS
}

\begin{abstract}
${ }^{1}$ Uno de los sacrificios más frecuentes y significativos del clínico es su orgullo. La vasta literatura sobre la divulgación de la contratransferencia (Blechner, 1992; Hirsch, 1995; Maroda, 1999) puede leerse como una historia del esfuerzo de "ir primero" a exponernos. Implícitamente no es diferente del pacto de la niñez "te mostraré los míos, si me muestras los tuyos". Sin conceptualizarlo de esta manera, siento que ofrecemos nuestro propio orgullo y creamos una atmósfera que conducirá a que el paciente se dé a conocer con la disposición de conocerse a sí mismo. Llamo el sacrificio del orgullo del clínico como que es "un tonto por amor", y lo veo como muy pertinente al tratamiento del narcisismo (ver capítulo 5 [Valores de la Clínica] para una discusión más completa de esto).

Los ejemplos de disposición de los clínicos de exponerse a sí mismos, incluye la discusión de Blechner, con un paciente, de su propio sueño (Blechner, 2001). La extensa casuística publicada de Maroda (1999, 2002) abunda en ilustraciones de su exposición de respuestas emocionales. Ehrenberg (1992) ha proporcionado también muchas viñetas pertinentes. El trabajo de Mitchell $(1993,1997)$ subraya el valor terapéutico potencial de la apertura del clínico. El concepto de Renik (1998) de auto-revelarse puede entenderse, desde esta perspectiva, tan efectiva, no tanto para qué se revela al paciente, como por los valores que actúa por estar dispuesto a exponerse a sí mismo.
\end{abstract}

Desde esta posición aventajada, podemos dialogar con las tendencias actuales en la literatura interpersonal y relacional, concordando en cuanto a lo que es efectivo, pero difiriendo en cuanto a por qué es efectivo: sin tener en cuenta, las razones para el revelarse en la mente del analista, lo que más afecta al paciente es que al revelarse el analista, expresa sus prioridades. Cuando ofrecemos decir al paciente nuestra experiencia, tomamos una posición acerca de lo que más cuenta en la vida. Manifestamos el valor de la salud sobre el del orgullo, el de tener impacto, sobre el de permanecer cómodo.

Anunciamos quién nos gustaría ser y lo mucho que nos esforzamos por hacer. Entre innumerables casos de esto, considero brevemente el trabajo de Coen (2002). En el material clínico que difunde su escrito, proporciona innumerables ejemplos de compartir su experiencia. Lo que los hace efectivos, no es tanto su contenido, sino más bien los valores que manifiestan. Para entender este punto, podría citar mucho del libro, pero permitiré que la viñeta siguiente sea suficiente (pp. 68-69):

El día anterior, otra vez habíamos experimentado su tendencia a destruir el significado y el valor del tratamiento. Una vez más élı insistió que debería terminar el tratamiento, y yo me sentí tentado a concordar con él. Pero, como pudimos hablar acerca de arruinar algo bueno entre nosotros, se pudo unir a mí y conjuntamente reflexionamos sobre lo que había sucedido en nuestra relación. Al día siguiente, al tratar de implicarlo acerca de lo que había añorado cuando era niño y ahora necesitaba, le conté sobre mi breve "conversación" telefónica, la tarde previa, con mi nieto de dos años en otra ciudad. Le dije que era muy triste que mi nieto pudiera esperar regularmente, conseguir lo que el Sr. N. había conseguido tan escasamente desde pequeño- padres, o yo como abuelo, gozando y admirándolo; en este ejemplo, mi nieto empieza a tener la habilidad de intercambiar unas pocas palabras por teléfono conmigo. El Sr. N. se conmovió por ambas cosas, por mi ejemplo y por compartir mi 
experiencia con él. Estaba especialmente conmovido porque yo no me quedé enojado y no fui rechazante con él por lo despectivo que había sido conmigo el día anterior.

Sugeriría que no es tanto lo que Coen compartió sino los valores que expresó, al escogerlos, lo que tuvo un impacto terapéutico significativo. Pienso en esto como un corolario del impacto limitado del insight. Así como el conocimiento de sí mismo del paciente, sólo puede ir hasta donde lo ayude a efectuar un cambio, así el contenido de la exposición de sí mismo del clínico, tiene menos importancia que los valores personificados en el relato. 